\title{
Statins Anti-Inflammatory Properties in Chronic Diseases
}

Mirto Nelso Prandini, Mahara B Nonato*, Anita LR Saldanha, Ana Paula M Pantoja, Andre L Gasparotto, Vitoria G Hernandes, Irina Antunes, Abel Pereira, Tereza L Bellincanta, Daphnne C Vera, Henrique AR Fonseca and Tania LR Martinez

BP-Hospital A Beneficiência Portuguesa de São Paulo, Brazil

*Corresponding author: Mahara B Nonato, BP-Hospital A Beneficiência Portuguesa de São Paulo, Brazil.
Received Date: September 24, 2019

Published Date: September 30, 2019

\begin{abstract}
Introduction Recently several clinical benefits of statins not directly related to the lipid-lowering action have been demonstrated. These are the so-called pleiotropic effects. Of great importance, some properties like anti-inflammatory activity and immunomodulatory actions of statins can be mentioned as well as the antithrombotic actions. Objectives We intended to demonstrate the role of statins in controlling some clinical manifestations in renal protection. Methods Ultra Sensitivity C-reactive Protein was chosen as the marker for inflammation. Results Patients on statins showed a decrease in CRP values, independent of lipid values, signaling to possible pleiotropic effect of the medication. Conclusion According to contemporary literature the therapeutic properties of statins may be amplified with new possibilities of its use in the treatment of several diseases : as rheumatic, renal or cardiac
\end{abstract}

Keywords: Statins; Inflammatory diseases; Pleiotropic effects

\section{Introduction}

Classically statins have been considered as lipid-lowering agents, selective and competitive inhibitors of 3-hydroxy-3-methylglutaryl-coenzyme-A reductase (HMG-CoA reductase), responsible for the conversion of HMG-CoA to mevalonate, a cholesterol precursor. Several studies [1-3] have shown that statins decrease plasma levels of cholesterol and lipoproteins by inhibiting HMGCoA reductase and cholesterol synthesis in the liver, increasing the number of LDL receptors on the surface of hepatocytes, with a consequent increase in absorption and catabolism of LDL. Recently, several clinical benefits of statins not directly related to the lipidlowering action have been demonstrated. These are the so-called pleiotropic effects $[4,5]$. Of great importance some properties like anti-inflammatory activity as well as immunomodulatory actions of statins can be mentioned.

\section{Objectives}

The main objective in the present study is demonstrate the role of statins in controlling some clinical manifestations in renal protection provided by its use. Ultra Sensitivity C-reactive Protein was chosen as the marker for inflammation.

\section{Methods}

Medline, Lilacs, Scielo and PubMed were searched for articles registered between 2000 and 2019 in English language, focused on trials demonstrating the statins anti-inflammatory properties in chronic inflammatory diseases, it's values and it's benefits. Inclusion criteria were trials that demonstrating the immunomodulatory actions with mensuration of LDL and C-reactive Protein. The exclusion criteria are those in which the studies comparing more than two chronic inflammatory diseases.

\section{Results and Discussion}

Patients on statins can show a decrease in CRP values, independent of lipid values, signaling to possible pleiotropic effect of the medication. The routinely use, also, is able to significantly improve flow-mediated vasodilation in patients with systemic lupus erythematosus (SLE), regardless the levels of cholesterol, suggesting its benefic use in patients with increased risk of developing early atherosclerotic disease, as is the case of patients with SLE. Treatment with atorvastatin in patients with systemic sclerosis resulted in an increase in bone marrow-derived endothelial 
precursor cells and an improvement in Raynaud's phenomenon. Renal protection by statins are not only due to cholesterol lowering but to pleiotropic effects as well. They are represented by inhibition of thrombosis, Inflammation and oxidative stress over all [6]. Experimental data point to normalizing renal values of oxidative stress demonstrated by urinary F2-isoprostane excretion and NADPH oxidase activity, protein expression of transforming growth factor TGF beta, fibronectin, mRNA expression of pro inflammatory molecules, oxidized LDL receptor1,endothelial NO synthase activity and protein expression, all of which are pleiotropic effect $[7,8]$.

As to clinical considerations, it is known that patients with chronic renal disease are at higher risk of cardiovascular events and that statins reduce cardiovascular mortality and several causes of mortality in the general population. Taking into consideration all clinical trials and meta-analysis the KDIGO guidelines on lipid management in adult patients with chronic renal disease a paradigm shift at proposals for statin use can be achieved: according to the patients cardiovascular risk it is possible to anticipate that statins may reduce it $[9,10]$. In addition to the antiinflammatory and antithrombotic actions of statins, its function as an immunomodulatory agent has also been described. Recently, the role of statins in the pathway of cell signaling mediated by isoprenylated proteins, especially geranyl-geranyl pyrophosphate (GGPP) and Rho-dependent protein system, has been highlighted. Statins inhibit the conversion of HMG-CoA reductase to mevalonate, which is involved in isoprenylation (post-translational modification) of GGPP and Rho system, which are proteins involved in cell maturation and division. By reducing the expression of these cellular signaling proteins, statins decrease the expression of tissue factor and cell proliferation factors $[11,12,13]$.

In endothelial cells statins inhibit the transcription of several $\mathrm{NF}-\mathrm{\kappa B}$ induced genes and decrease interferon-g induced class II HLA expression. The suppression of the major histocompatibility complex of class II (MHCII) precludes the activation of $\mathrm{T}$ cells and can modulate Th-type response-dependent autoimmunity $[12,13]$. Thus, there are studies showing the use of statin in organ transplants and other conditions requiring immunosuppression. Treatment with pravastatin associated with immunosuppressive drugs, such as ciclosporin, prednisone and azathioprine in patients with heart transplantation, significantly reduced the frequency of rejection, increasing the survival of these patients when compared to patients receiving immunosuppressants, without the statin [13].

The demonstration of the multiple effects of statins has justified some studies evaluating the role of statins in controlling the clinical manifestations of some systemic autoimmune diseases. An experimental study evaluated the effect of simvastatin on collagen-induced arthritis in mice, which is dependent on Th-1 type response. In this study [14], with the use of simvastatin prior to the induction of arthritis, it was observed that the mice demonstrated a lower inflammatory process than controls and, when used after the development of arthritis, they showed an improvement in the inflammatory process. This effect was observed with the use of simvastatin in doses insufficient to alter cholesterol concentrations. Cellular and humoral immune responses of Th-1 type were suppressed significantly in ex vivo analysis.

McCarey et al. [15] carried out a clinical study demonstrating that patients with rheumatoid arthritis who received 40mg / day of atorvastatin during six months showed improvement of DAS28 (score of disease activity) when compared with the placebo group.

Steffens \& Mach [16] demonstrated that the use of atorvastatin for 14 days in F1 NZB / W mice (experimental model of systemic lupus erythematosus) was able to reduce serum levels of anti-DNA IgG antibodies, improve proteinuria and reduce immunoglobulin deposits in the renal glomeruli. Significant reduction of MHC class II expression was also observed in target organs, such as spleen and kidney.

\section{Conclusion}

In summary, many studies suggest that the therapeutic properties of statins may be amplified. A new possibility of its use refers to its application in the treatment of several chronic inflammatory diseases, particularly chronic renal disease patients, regardless of the lipid-lowering effect. We believe, however, that further studies should be performed to prove its benefit in such conditions not directly related to dyslipidemias.

\section{Acknsowledgement}

None.

\section{Conflict of Interest}

No conflict of interest.

\section{References}

1. Koh KK (2000) Effects of statins on vascular wall: vasomotor function, inflammation, and plaque stability. Cardiovasc Res 47(4): 648-657.

2. Davidson MH, Toth PP (2004) Comparative effects of lipid-lowering therapies. Prog Cardiovasc Dis 47: 73-104.

3. Liao, James K (2005) Clinical implications for statin pleiotropy. Curr Opin Lipidol 16: 624-629.

4. Campo Vanessa Leiria, Carvalho Ivone (2007) Hypolipemic statins and new therapeutcal trends. Quím Nova São Paulo 30(2): 425-430.

5. Meroni PL, Luzzana C, Ventura D (2002) Anti-inflammatory and immunomodulating properties of statins. An additional tool for the therapeutic approach of systemic autoimmune disease. Clin Rev Allerg Immunol 23: 263-277.

6. Faludi AA, Izar MCO, Saraiva JFK, Chacra APM, Bianco HT, et al. (2017) Atualização da Diretriz Brasileira de Dislipidemias e Prevenção da Aterosclerose - 2017. Arq Bras Cardiol 109(2Supl.1): 1-76.

7. Zhou MS, Schuman IH, Jaimes EA, Raij L (2008) Renoprotection by statins is linked to a decrease in oxidative stress, TGF-beta, and fibronectin with concomitant increase in nitric oxide bioavailability. Am J Physiol Renal Physiol 295(1): F53-9.

8. Palaniswamy C, Selvaraj DR, Selvaraj T, Sukhija R (2010) Mechanisms underlying pleiotropic effects of statins. Am J Ther 17(1): 75-78.

9. Oesterle A, Laufs U, Liao JK (2017) Pleiotropic Effects of Statins on the Cardiovascular System. Circ Research 120(1): 229-243.

10. Schneider MP, Hübner S, Titze SI, Schmid M, Nadal J, et al. (2015) Implementation of the KDIGO guideline on lipid management requires a substantial increase in statin prescription rates. Kidney Int 88(6): 14111418. 
11. Jaiswal SR, Sontakke SD (2012) Experimental evaluation of analgesic and anti-inflammatory activity of simvastatin and atorvastatin. Indian Pharmacol 44(4): 475-479.

12. Serrano Jr CV, Ascer E, Venturinelli M (2001) The effect of atorvastatin on proinflammatory mediators and endothelial adhesion molecule expression. Eur Heart J 22: 646.

13. Mehra MR, Uber PA, Vivekananthan K, Solis S, Scott RL, et al. (2002) Comparative beneficial effects of simvastatin and pravastatin on cardiac allograft rejection and survival. J Am Coll Cardiol 40: 1609-1614.
14. Leung BP, Sattar N, Crilly A, Prach M, McCarey DW, et al. (2003) A novel anti-inflammatory role for sinvastatin in inflammatory arthritis. J Immmunol 170(3): 1524-1530.

15. McCarey DW, McInnes IB, Madhok R, Hampson R, Scherbakov O, et al. (2004) Trial of atorvastatin in rheumatoid arthritis (TARA): doubleblind, randomized placebo-controlled trial. The Lancet 363: 2015-2021.

16. Steffens S, Mach F (2006) Drug insight: Immunomodulatory effects of statins--potential benefits for renal patients. Nat Clin Pract Nephrol 2(7): 378-387. 